\title{
Evaluación económica de los servicios ecosistémicos del recurso vegetal de la parroquia Punin, cantón Riobamba
}

DOI: https://doi.org/10.33262/ap.v3i3.1.87

\begin{abstract}
(c) (1) (2)
Economic evaluation of the ecosystem services of the plant resource of the parroquia Punin, canton Riobamba

Marcela Yolanda Brito Mancero. ${ }^{1}$, Landy Elizabeth Ruiz Mancero. ${ }^{2}$ \& Karina Viviana Lemache Velarde. ${ }^{3}$
\end{abstract}

\begin{abstract}
.
Introduction: The valuation of ecosystem services is not new according to the Food and Agriculture Organization of the United Nations, it considers that ecosystem goods can be valued at 125 trillion USD, their maintenance or degradation has an impact on gains or losses of values due to what is required to perform analysis and evaluations for development decision making. Punín is one of the rural parishes of the Riobamba canton, its population amounts to 5,976 people and is divided into 23 communities that are mostly recognized as indigenous, the productive economic activities are agriculture and livestock, a large part of the production is commercialized and other part is used for selfconsumption. Objective: To economically estimate the ecosystem services of the productive plant resource. Methodology: An analysis of the perception of environmental services and the willingness to pay for the conservation of the plant resource was carried out through the application of 359 in 8 communities selected by the criteria of number of inhabitants and agricultural production area, the inhabitants ranked the Ecosystem services using a Likert scale, the sociodemographic variables that affect the perception of

\footnotetext{
${ }^{1}$ Escuela Superior Politécnica de Chimborazo, Facultad de Recursos Naturales. Riobamba, Ecuador. mybrito@espoch.edu.ec, https://orcid.org/0000-0003-2689-3516

${ }^{2}$ Escuela Superior Politécnica de Chimborazo, Facultad de Informática y Electrónica. Riobamba, Ecuador. lruiz@espoch.edu.ec, https://orcid.org/0000-0003-1818-1084

${ }^{3}$ Investigador independiente en el área, karilv90@ hotmail.com, https://orcid.org/0000-0002-7828-6331.
} 
environmental services were analyzed and the economic value of the resource was estimated using the contingent method. Results: The most valued ecosystem services are provisioning services, followed by support and regulation services that classify them as very important and cultural services as important. The age variable affects the perception of supporting ecosystem services. The calculated DAP is 5,393.63 USD / year, the value of direct use of the productive plant resource was established at 4,038,539.60 USD / year and the total economic value through the contingent method amounts to 4043933.23 USD / year. Conclusions: The variable level of education significantly influences the perception of support services, possibly because this category of services is the most complex to distinguish. The TEV of the productive plant resource calculated using the contingent method can vary according to the demand for the good in the market and the main products of the parish commercialization such as vegetables and tubers present constant price fluctuations in the market

Keywords: Ecosystem services, environmental services, economic valuation, environmental perception, parroquia Punín.

\section{Resumen.}

Introducción: La valoración de los servicios ecosistémicos no es nueva según la Organización de las Naciones Unidas para la Alimentación y la Agricultura, considera que los bienes ecosistémicos pueden valorarse en 125 billones de USD, su mantenimiento o degradación repercuten en ganancias o pérdidas de valores por lo que se requiere realizar análisis y evaluaciones para la toma de decisiones de desarrollo. Punín es una de las parroquias rurales del cantón Riobamba su población asciende a 5976 personas y se divide en 23 comunidades que se reconocen en su mayoría como indígenas, las actividades económicas productivas son la agricultura y la ganadería gran parte de la producción se comercializa y otra parte es utilizada en el autoconsumo. Objetivo: Estimar económicamente los servicios ecosistémicos del recurso vegetal productivo. Metodología: Se realizó un análisis de la percepción de los servicios ambientales y la disposición a pagar por la conservación del recurso vegetal mediante la aplicación de 359 en 8 comunidades seleccionadas mediante los criterios de número de habitantes y área de producción agrícola, los habitantes jerarquizaron los servicios ecosistémicos utilizando una escala de Likert, se analizó las variables sociodemográficas que inciden en la percepción de los servicios ambientales y se estimó el valor económico del recurso utilizando el método contingente. Resultados: Los servicios ecosistémicos más apreciados son los de aprovisionamiento, seguidos por servicios de soporte y regulación que los catalogan como muy importante y los servicios culturales como importante. La variable edad incide en la percepción de los servicios ecosistémicos de soporte. El DAP calculado es de 5393,63 USD/año, el valor de uso directo del recurso vegetal de productivo se estableció en 4038539,60 USD/año y el Valor económico total mediante el método contingente asciende a 4043933,23 USD/año. Conclusiones: La variable nivel de educación influye significativamente en la percepción que presentan los servicios de soporte posiblemente porque esta categoría de servicios es la más compleja de distinguir. 
La VET del recurso vegetal productivo calculada mediante el método contingente puede variar según la demanda del bien en el mercado y los principales productos de comercialización de la parroquia como las hortalizas y tubérculos presentan fluctuaciones constantes de precios en el mercado.

Palabras claves: Servicios ecosistémicos, servicios ambientales, valoración económica, percepción ambiental, parroquia Punín.

\section{Introducción.}

La Clasificación Internacional Común de Servicios de los Ecosistemas reconoce que las principales categorías de bienes y servicios de los ecosistemas son los servicios de aprovisionamiento, de regulación y culturales (Haines-Young \& Potschin-Young, 2018); los cuales al proveer alimentos y agua para el consumo, energía y control microclimas, permitir acciones como la polinización y formación de suelos y al ofrecer beneficios recreativos permiten el desarrollo de la vida humana. (Life adaptamed, 2018)

La valoración de los servicios ecosistémicos no es nueva según la Organización de las Naciones Unidas para la Alimentación y la Agricultura, considera que los bienes ecosistémicos pueden llegar a valorarse en 125 billones de USD pero que no han recibido el cuidado necesario en el ámbito económico, ambiental ni legal. (FAO, 2021). El mantenimiento o degradación de los recursos naturales repercuten en ganancias o pérdidas de valores por lo que se requiere realizar análisis y evaluaciones como instrumentos para la toma de decisiones complejas que exige el cuidado de los recursos naturales. (Barbier, Acreman, \& Knowler, 1997).

El Recurso vegetal presta variados beneficios sociales como la regulación de procesos micro climáticos, provisión de alimentos y medicinas, y el uso en aspectos espirituales y culturales de la población los cuales no siempre son percibidos o reconocidos por los pobladores y autoridades. Aunque no todo son efectos son positivos, también se puede advertir algunas problemáticas como en el caso de la agricultura, en la búsqueda del incremento de la productividad con el incorrecto uso de plaguicidas puede conllevar la disminución de la polinización natural, y afecciones al recurso suelo, la deforestación aumenta el riesgo de deslizamientos e inundaciones. (Lemache, 2021)

Punín es una de las parroquias rurales del cantón Riobamba su población asciende a 5976 personas y se divide en 23 comunidades de las cuales 22 se reconocen como indígenas y la cabecera parroquial como mestiza. (INEC, 2021).

Las actividades económicas productivas son la agricultura y la ganadería, la agricultura es muy diversa entre los principales cultivos existen gramíneas tales como maíz, cebada, vicia, trigo, avena, hortalizas como el haba, las papas, el tomate riñón, el pimiento, frutas por ejemplo mora y frutilla y productos andinos como quinua, chochos, oca y melloco. (GADPR PUNÍN, 2015) La mayor parte de la producción está dedicada a la 
comercialización y otra parte para el autoconsumo. En lo referente a la ganadería existe una gran variedad de especies pecuarias como el ganado ovino, porcinos, aves y especies menores como cuyes y conejos que generalmente se lleva cabo de forma extensiva, se establecen en los patios de las casas y su abono es utilizado directamente las parcelas de tierra sin previo tratamiento generalmente. (Rodriguez, Herrera, \& Ruiz, 2014)

\section{Metodologia.}

\section{Área de estudio.}

La parroquia rural de Punín cuenta con una población es de 5976 habitantes (INEC, 2001), divididas en 23 comunidades, se ubica a $12 \mathrm{Km}$ al Sur de la ciudad de Riobamba en la vía a Macas, tiene una extensión de $52 \mathrm{Km} 2$, limita al norte con la parroquia San Luis, al sur con la parroquia Flores, al este con la quebrada Guaslán de parroquia Licto y al oeste limita el cantón Colta. La altitud media de la parroquia es 2.800 m.s.n.m. su área se divide en tres zonas, zona baja que va de 2.720 a 2.950 m.s.n.m.; la zona media de 2.900 a 3.380 m.s.n.m. y zona alta de 3.400 a 3.500 m.s.n.m. (GADPR PUNÍN, 2015).

Figura 1

Límites de la parroquia rural de Punín

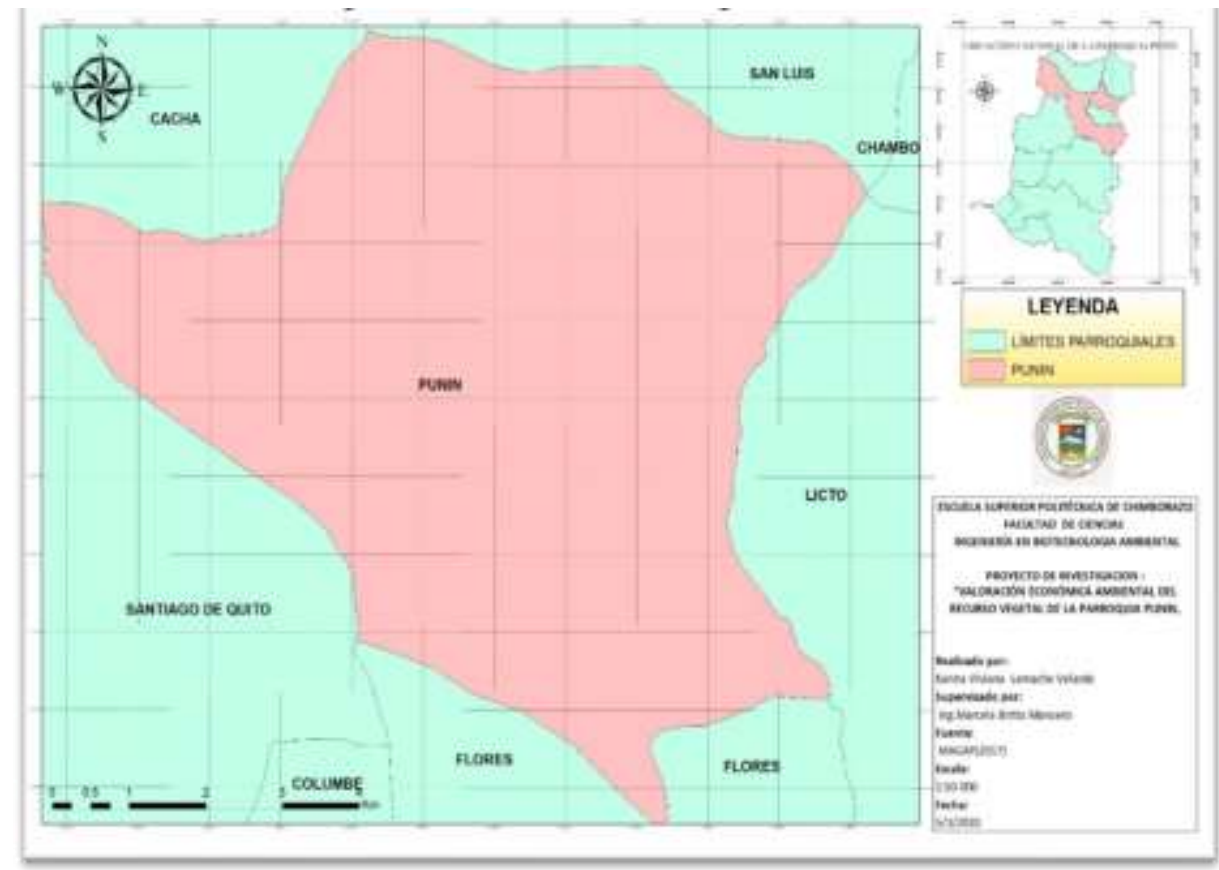

Elaboración: Los Autores

Según la clasificación ecológica gran parte del territorio de Punin se ubica como bosque seco - Montano Bajo (bs-MB) y las formaciones ecológicas de la zona media se encuentra en la zona de vida estepa espinoso montana Baja (eeMB). En lo referente al clima las temperaturas que van de $12^{\circ} \mathrm{C}$ a $18^{\circ} \mathrm{C}$, con una precipitación media de $500 \mathrm{~mm}$ anuales y una velocidad de viento promedio de 4m/s. (GADPR PUNÍN, 2015). 


\section{Selección de muestra}

Para determinar la selección de la muestra se consideró las comunidades con mayor superficie de suelos cultivados, disponibilidad de recursos, facilidad del acceso y número de habitantes por comunidad por lo que se seleccionaron 8 de las 24 comunidades que posee la parroquia, para la selección del tamaño de la población se utilizó la fórmula de Canavos (1998), el número de encuestas aplicadas para a los 5976 habitantes fue de 359 se detalla en la tabla 1 .

\section{Tabla 1}

Distribución de encuestas por comunidad

\begin{tabular}{ccc}
\hline No & Comunidad & No. Encuestas \\
\hline 1 & Cabecera Parroquial & 43 \\
2 & Gulalag & 30 \\
3 & San Antonio de Bashalan & 23 \\
4 & San Isidro & 105 \\
5 & San Pedro de Pulshi & 23 \\
6 & Santa Bárbara & 42 \\
7 & Siguilan & 57 \\
8 & Tzalaron & 36 \\
\hline & TOTAL & $\mathbf{3 5 9}$ \\
\hline
\end{tabular}

Fuente: (Lemache, 2021)

\section{Colección de los datos}

Las fuentes de información para el presente trabajo fue la revisión bibliográfica del plan de ordenamiento territorial de la parroquia Punin, la información del INEC, consulta a las partes interesadas identificadas que fueron: el presidente de la junta parroquial, los moradores de las comunidades y funcionarios del ministerio de Agricultura; y encuestas a los pobladores de las comunidades seleccionadas.

Los datos utilizados tienen dos enfoques, el cuantitativo que proviene de la aplicación de encuestas y levantamiento de la información de costos de producción del recurso vegetal mediante la información del sistema único de información agropecuaria para 2020 y un enfoque cualitativo resultante de la caracterización de los servicios ecosistémicos brindados por el recurso vegetal del sitio en análisis.

\section{Clasificación de los servicios ecosistémicos}

La identificación y jerarquización de los servicios ecosistémicos se la realizó mediante la aplicación de encuestas donde se utilizó una escala de Likert donde los habitantes encuestados evaluaron los servicios utilizando los criterios detallados en la tabla 2.

También se relaciona las variables socioeconómicas que inciden en la percepción de los servicios ambientales del recurso vegetal 
Tabla 2

Escala de valoración de los servicios ecosistémicos.

\begin{tabular}{lccccc}
\hline CRITERIO & $\begin{array}{c}\text { No me } \\
\text { llama la } \\
\text { atención }\end{array}$ & $\begin{array}{c}\text { Nada } \\
\text { importante }\end{array}$ & $\begin{array}{c}\text { Poco } \\
\text { importante }\end{array}$ & Importante & $\begin{array}{c}\text { Muy } \\
\text { importante }\end{array}$ \\
\hline ESCALA & $0-2$ & $2-4$ & $4-6$ & $6-8$ & $8-10$ \\
\hline \multicolumn{5}{c}{ Fuente: (Lemache, 2021) }
\end{tabular}

\section{Valoración económica}

Para establecer el Valor económico total (VET) se realizó el análisis de los componentes del valor de uso y no uso, mediante la estimación del valor total de los bienes y servicios que provee el recurso vegetal y la disposición a pagar (DAP) por la conservación y el manejo del recurso vegetal productivo en la parroquia. (Baral, Basnyat, Khanal, \& Gauli, 2016)

\section{Resultados.}

Estimación ambiental de los servicios ecosistémicos del recurso vegetal

Tabla 3

Valores medios de los servicios ecosistémico estimado por comunidad.

\begin{tabular}{|c|c|c|c|c|c|c|c|c|}
\hline \multirow[b]{2}{*}{ Comunidades } & \multicolumn{2}{|c|}{ SA } & \multicolumn{2}{|c|}{ SR } & \multicolumn{2}{|c|}{ SS } & \multicolumn{2}{|r|}{ SC } \\
\hline & 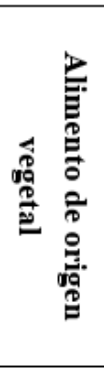 & 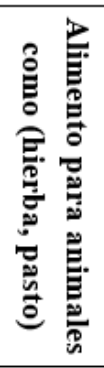 & 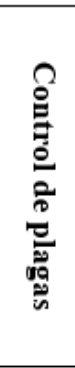 & 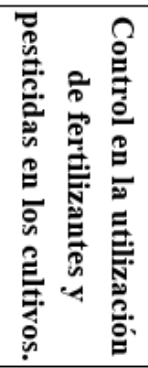 & 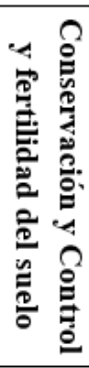 & 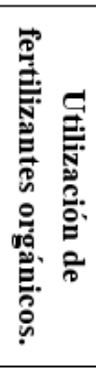 & 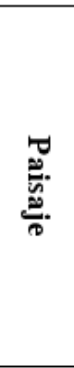 & 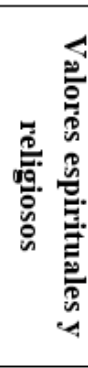 \\
\hline SAN ISIDRO & 8,1 & 8 & 8 & 8,1 & 7,9 & 8,6 & 8 & 8,1 \\
\hline SIGUILAN & 8,5 & 8 & 8,8 & 8,2 & 8 & 7,5 & 8 & 7,9 \\
\hline $\begin{array}{c}\text { CABECERA } \\
\text { PARROQUIAL }\end{array}$ & $* 9$ & 7,7 & 7,8 & 8,2 & 8,2 & 8,2 & 6,9 & 7,6 \\
\hline SANTA BÁRBARA & 8,9 & 8 & 8,8 & 8 & 7,9 & 7,1 & 7,8 & 7,2 \\
\hline TZALARON & 8,1 & 8,9 & 7,8 & 7,7 & 7,6 & 6,4 & 7,2 & 7,4 \\
\hline GULALAG & 8,3 & 8,8 & 8,4 & 7,4 & 7,4 & 8,1 & 7,7 & 7,4 \\
\hline $\begin{array}{l}\text { SAN PEDRO DE } \\
\text { PULSHI }\end{array}$ & 7,9 & 8,6 & 8,2 & 6,9 & 8,1 & 7,4 & 7,3 & 7,4 \\
\hline $\begin{array}{c}\text { SAN ANTONIO DE } \\
\text { BASHALAN }\end{array}$ & 8,8 & 8,8 & 8,8 & 7,1 & 7,1 & 6,8 & $* 7,0$ & 7,9 \\
\hline $\begin{array}{c}\text { PROMEDIO POR } \\
\text { SERVICIO }\end{array}$ & 8,45 & 8,35 & 8,32 & 7,7 & 7,78 & 7,51 & 7,5 & 7,6 \\
\hline
\end{tabular}

Fuente: (Lemache, 2021) 


\section{Gráfico 1}

Promedios ponderados por servicios ecosistémicos

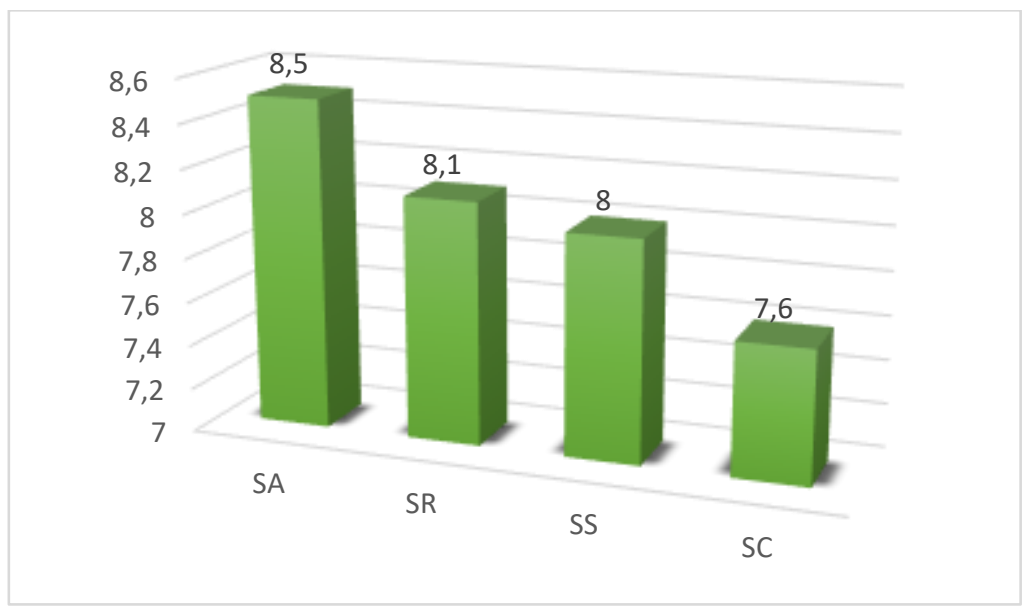

Fuente: (Lemache, 2021)

En la tabla 3 y gráfica 1 se puede observar que la apreciación de los servicios ecosistémicos sigue el siguiente orden: servicios de abastecimiento, servicios de regulación, servicios de soporte y finalmente servicios culturales, lo que se asemeja a resultados anteriores en donde han encontrado que la preferencia de los SE están orientan a los servicios de provisión, seguidos por los de regulación y culturales (Agbenyega, Burgess, Cook, \& J., 2009).

en los pobladores de la parroquia de Punín tienen una alta percepción sobre servicios ecosistémicos brindados por el recurso vegetal esto confirma lo dicho por (MartínLópez, y otros, 2021) que establece que las poblaciones rurales identifican con mayor frecuencia los servicios de abastecimiento, siendo este el servicio mejor puntuado en la cabecera cantonal el abastecimiento en provisión de alimentos de origen vegetal con 9 que corresponde a muy importante, según lo manifestado por los pobladores esto se debe a que la producción de alimentos está destinada a la comercialización y cierta parte para el autoconsumo, además que la agricultura y la ganadería son las actividades económicas de la mayor parte de los encuestados, lo que coincide con estudios similares realizados en la provincia de Chimborazo y el cantón Riobamba lo señalado por (Moreta, 2018) (Carrasco, 2019) (Mayanquer, 2019) (Collaguazo, 2019). Por otra parte los servicios culturales obtuvieron la menor puntuación, en San Antonio de Bashalan el servicio de paisaje obtuvo una estimación de 7 que en la escala evaluada corresponde a importante, esto podría explicarse por qué los servicios culturales son los menos tangibles según lo establece (Daniel, y otros, 2012).

\section{Relación de las características sociodemográficas con la percepción de los servicios} ecosistémicos 
Tabla 4

Características sociodemográficas de la población encuestada en la parroquia Punín

\begin{tabular}{|c|c|c|}
\hline Características & Variables & Porcentaje \\
\hline \multirow{2}{*}{ Género } & Hombre & $52,33 \%$ \\
\hline & Mujer & $47,67 \%$ \\
\hline \multirow[t]{5}{*}{ Estado Civil } & Soltero & $21,09 \%$ \\
\hline & Unión libre & $5,21 \%$ \\
\hline & Casado & $56,16 \%$ \\
\hline & Divorciado & $3,86 \%$ \\
\hline & Viudo & $13,70 \%$ \\
\hline \multirow[t]{3}{*}{ Edad } & Menor a 30 años & $21,37 \%$ \\
\hline & $31-60$ años & $49,03 \%$ \\
\hline & Mayor 60 años & $29,60 \%$ \\
\hline \multirow[t]{6}{*}{ Nivel de Educación } & Primaria incompleta & $42,47 \%$ \\
\hline & Primaria completa & $19,45 \%$ \\
\hline & Secundaria incompleta & $22,46 \%$ \\
\hline & Secundaria completa & $11,51 \%$ \\
\hline & Tercer nivel incompleto & $2,47 \%$ \\
\hline & Tercer nivel completo & $1,64 \%$ \\
\hline \multirow[t]{6}{*}{ Ocupación } & Ama de casa & $18,90 \%$ \\
\hline & Empleado & $20,82 \%$ \\
\hline & Independiente & $45,21 \%$ \\
\hline & desempleado & $5,21 \%$ \\
\hline & Estudiante & $6,85 \%$ \\
\hline & Jubilado & $3,01 \%$ \\
\hline \multirow[t]{4}{*}{ Ingresos } & Menor a 394 dólares & $79,18 \%$ \\
\hline & 394 - 788 dólares & $15,33 \%$ \\
\hline & 778 a 1182 dólares & $4,94 \%$ \\
\hline & 1000 a 2000 dólares & $0,55 \%$ \\
\hline
\end{tabular}

Fuente: (Lemache, 2021)

Tabla 5:

Variables sociodemográficas que inciden en la apreciación de los servicios ecosistémicos.

\begin{tabular}{lrrrr}
\hline \multicolumn{1}{c}{ VARIABLES } & \multicolumn{1}{c}{ SA } & \multicolumn{1}{c}{ SR } & \multicolumn{1}{c}{ SS } & \multicolumn{1}{c}{ SC } \\
\hline GÉNERO & 0,494161407 & 0,0679139 & 0,12646346 & 0,71003418 \\
EDAD & 0,226413711 & 0,844651885 & 0,080610946 & 0,533298735 \\
ESTADO CIVÍL & 0,478803102 & 0,95142534 & 0,201805247 & 0,715504718 \\
NIVEL DE & 0,826297308 & 0,581506384 & $\mathbf{0 , 0 2 5 2 3 0 8 1 3} *$ & 0,062830655 \\
EDUCACIÓN & & & & \\
INGRESOS & 0,884807128 & 0,221386125 & 0,166306905 & 0,195929009 \\
MENSUALES & & & & \\
OCUPACIÓN & 0,909401313 & 0,375177373 & 0,375177373 & 0,310836668 \\
\hline
\end{tabular}

${ }^{*}$ Valor de $\mathrm{P}<0,05$

Fuente: (Lemache, 2021) 
La relación entre la variable nivel de educación con respecto a los servicios ecosistémicos de soporte presentan una inferencia significativa en comparación a relación del resto de variables con los servicios de abastecimiento, regulación y culturales esto se puede explicar por lo establecido por (Poppenborg \& Koellner, 2013) quienes indican que la educación presenta una influencia positiva en relación a la comprensión de los servicios de regulación y apoyo.

\section{Valor de uso directo recurso vegetal productivo}

- Valoración del recurso vegetal productivo

Tabla 6

Producción del recurso vegetal parroquia Punín.

\begin{tabular}{|c|c|c|c|c|c|c|c|c|c|}
\hline \multirow[t]{2}{*}{ Cultrvo } & $\frac{y}{\frac{y}{2}}$ & \multirow[t]{2}{*}{$\begin{array}{l}\frac{8}{E} \\
\text { है } \\
\text { है }\end{array}$} & है & $\frac{8}{8}$ & 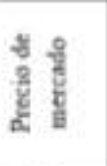 & $\frac{8}{\text { ă }}$ & $\begin{array}{l}\frac{2}{2} \\
\frac{g}{g} \\
\frac{8}{8} \\
\frac{8}{8} \\
\text { है }\end{array}$ & $\frac{\mathrm{g}}{\mathrm{g}} \mathrm{g}$ & 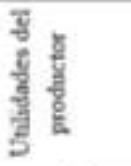 \\
\hline & (Ha) & & (Ton/Ha) & (Ton) & (5) Ton & (5) & SHa & (5) & (5) \\
\hline MU/Z & 148,84 & 15,2 & 10,00 & 1488,40 & 520,00 & 773968,00 & 950,00 & 141398,00 & 632570,00 \\
\hline$P A P A$ & 107,23 & 11 & 13,20 & 1415,96 & 650,00 & 920376,60 & 2620,00 & 281047,40 & 639329,20 \\
\hline HABA & 104,01 & 10,6 & 3,81 & 396,25 & 420,00 & 166436,80 & 500,00 & 83200,00 & 83228,80 \\
\hline LECHUGA & 114,26 & 11.7 & 6,67 & 762,11 & 356,00 & 271312,66 & 3270,00 & 373630,20 & $-102317,54$ \\
\hline ncu & 76,98 & 7,86 & 3.21 & 247.11 & 120,00 & 29652,70 & 560,00 & 43108,80 & $-13456,10$ \\
\hline PASTO & 36,35 & 3,71 & 11,00 & 399,85 & 125,00 & 49981,25 & 780,00 & 28353,00 & 21628,25 \\
\hline $\begin{array}{l}\text { TOMATE } \\
\text { RLNON }\end{array}$ & 29,64 & 3,02 & 145,50 & 4312,62 & 670,00 & 2859455,40 & 16750,00 & 496470,00 & 2392985,40 \\
\hline ALFALFA & 29.26 & 2,99 & 17,35 & 507.66 & 567.00 & 257543,79 & 360,00 & 10533,60 & 277310,19 \\
\hline ARVEJA & 14,32 & 1.46 & 3,00 & 42,96 & 430,00 & 18472,80 & 1443,00 & 20663,76 & $-2190,96$ \\
\hline FRLTIILA & 12,25 & 1.25 & 3,42 & 41,90 & 1200,00 & 50274,00 & 1570,00 & 19232,50 & 31041,50 \\
\hline QUINUA & 87,05 & 8,89 & 0,90 & 78,35 & 850,00 & 66593,25 & 900,00 & 78345,00 & $-11751,75$ \\
\hline $\mathrm{CHOCHO}$ & 27,96 & 2,86 & 1,14 & $31,8 ?$ & 430,00 & 13705,99 & 1320,00 & 36907,20 & $-23201,21$ \\
\hline$O C A$ & 18,64 & 1,91 & 0,75 & 13,98 & 96,00 & 1342,08 & 864,00 & 16104,96 & $-14762,88$ \\
\hline MEILOCO & 18,64 & 1,91 & 0,45 & 8,39 & 430,00 & 3606,84 & 652,00 & 12153,28 & $-8546,44$ \\
\hline FRENOL & 15.01 & 1,53 & 1,00 & 15,01 & 500,00 & 7505,00 & 1200,00 & 18012,00 & $-10507,00$ \\
\hline MORA & 3,98 & 0,41 & 8.00 & 31.84 & 1340,00 & 42665,60 & 2800,00 & 11144,00 & 31521,60 \\
\hline CEBADA & 87,98 & 8,99 & 2,05 & 180,36 & 340,00 & 97393,86 & 780,00 & 68624,40 & 28769,46 \\
\hline IRIGO & 25,16 & 2,57 & 1,82 & 45,79 & 476,00 & 21796,61 & 780,00 & 19624,80 & 2171,81 \\
\hline AVENA & 20,69 & 2,11 & 5,57 & 115,24 & 780,00 & 89889,77 & 250,00 & 5172,50 & 84717,27 \\
\hline TOTAL & 978,29 & 100 & & & & & & & 4038539,60 \\
\hline
\end{tabular}

Fuente: (GADPR PUNÍN, 2015) (SIPA, 2020)

Elaboración: (Lemache, 2021)

En el sistema de Información pública agropecuaria se puedo obtener los valores comerciales de productos convencionales tales como la papa, el maíz, el tomate, entre otros; mientras que productos dedicados en mayor parte al autoconsumo como la oca y la vicia no constan el portal de información por lo que fue necesario recurrir a los precios directos de mercado.

Dentro del cuadro de producción se puede analizar que el tomate riñón es el cultivo que mayor utilidad representa a los productores, este dato se asemeja a lo establecido en 
estudios similares realizado en las parroquias rurales del cantón Riobamba donde indican que el tomate riñón es el cultivo más rentable (Collaguazo, 2019), seguido por la papa y el maíz. Los cultivos andinos como chocho, quinua, melloco y oca se encuentran dentro de los productos en que la utilidad es negativa esto puede estar sujeto a que estos cultivos por tradición han formado parte del sistema agrícola de los productores de auto subsistencia y que generalmente al mercado llegan los excedentes. (Nieto, y otros, 2016)

- Disposición a pagar por la conservación del recurso vegetal

Tabla 7: Disposición a pagar.

\begin{tabular}{lllll}
\hline DAP & HABITANTES & PROMEDIO & NÚMERO DE & DAP TOTAL \\
USD/AÑO & & (Hab/familia) & USUARIOS & USD/AÑO
\end{tabular}

\begin{tabular}{lllll}
\hline 10,64 & 3932 & 3,7 & 1063 & 5393,63 \\
\hline
\end{tabular}

Fuente: (Lemache, 2021)

Gráfico 2

Disposición a pagar por la conservación del recurso vegetal

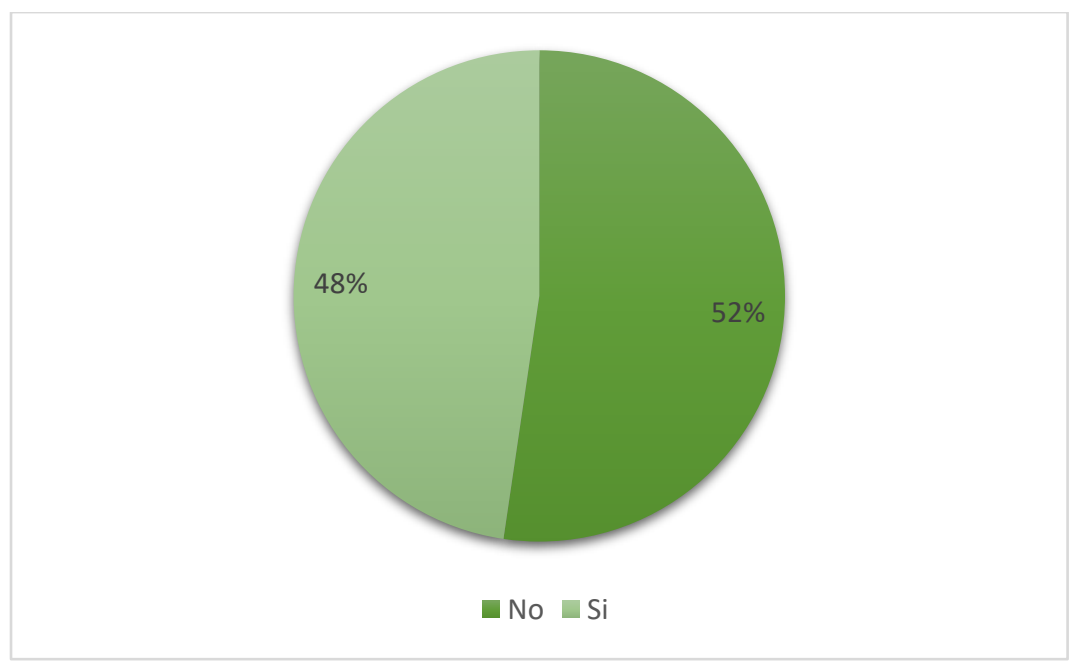

Fuente: (Lemache, 2021)

En cuanto a la DAP el $48 \%$ de la población dispuesta a pagar contribuirían con un valor de 10,64 UDS al año lo que resultaría un valor de 5393,63 USD al año. En lo referente a la población que no está dispuesta a pagar el 52,33\% indica que la principal razón indica que a que su bajo nivel de ingreso limitante que esta señalada en el documento Métodos de valoración económica de los servicios ambientales. (Cristeche \& Penna, 2008).

- Valor económico total (VET) 
Tabla 8

Cálculo de la valoración económica del recurso vegetal por el método contingente.

\begin{tabular}{lc}
\hline \multicolumn{1}{c}{ MÉTODO DE VALORACIÓN } & USD ANUAL \\
\hline $\begin{array}{l}\text { VALOR DE USO DIRECTO (Valor del recurso vegetal } \\
\text { productivo) }\end{array}$ & 4038539,597 \\
& \\
\hline DAP ANUAL & 5393,63 \\
\hline VET & $\mathbf{4 0 4 3 9 3 3 , 2 2 7}$ \\
\hline
\end{tabular}

Fuente: (Lemache, 2021)

El cálculo del VET por el método contingente permite calcular el valor total del bien empleando datos de valor de uso del recurso vegetal productivo que esta en función de la demanda del bien en el mercado (Cristeche \& Penna, 2008) que en este caso es muy fluctuante por lo que estos datos pueden estar sujetos a la variación del mercado. El recurso vegetal productivo de la parroquia Punín se calcula en 4043933,227 USD al año el cual debería destinarse a actividades de tanto de conservación como de recuperación del recurso (Laurans, Rankovic, Billé, Pirard, \& Mermet, 2013) proponen que el valor económico total de los bienes y servicios deben utilizarse en propósitos relevantes a implementación de políticas informativas, y técnicas de conservación de los recursos.

\section{Conclusiones.}

- La población de la parroquia rural de Punín muestra una buena percepción de los servicios ecosistémicos del recurso productivo vegetal, el orden de jerarquización de los mismos fue en primer lugar los servicios de abastecimiento, seguido por los servicios de regulación, soporte que los catalogaron como muy importante y finalmente los servicios culturales fueron clasificados como importantes.

- La variable nivel de educación influye significativamente en la percepción que presentan los servicios de soporte posiblemente porque esta categoría de servicios es la más compleja de distinguir.

- La valoración económica del recurso vegetal productivo calculada mediante el método contingente puede variar según la demanda del bien en el mercado y los principales productos de comercialización de la parroquia como las hortalizas y tubérculos presentan fluctuaciones constantes de precios en el mercado, por lo que el VET puede verse modificado por esta variable.

- La principal razón por la cual los pobladores de la parroquia Punín no presentan disposición a pagar por la conservación del recurso se debe a que sus ingresos son muy limitados.

\section{Referencias}

Agbenyega, O., Burgess, P., Cook, M., \& J., M. (2009). Application of an ecosystem function framework to perceptions of community woodlands. Land Use Policy, 551 - 557. doi:https://doi.org/10.1016/j.landusepol.2008.08.011. 
Baral, S., Basnyat, B., Khanal, R., \& Gauli, K. (2016). A Total Economic Valuation of Wetland Ecosystem Services: An Evidence from Jagadishpur Ramsar Site, Nepal. Scientific World Journal. doi:https://doi.org/10.1155/2016/2605609

Barbier, E., Acreman, M., \& Knowler, D. (1997). Economic valuation of Wetlands. Ramsar convention Bureau department of environmental economics and environmental management, University of York institute of hydrology IUCN-the world conservation union, Switzerland.

Carrasco, D. (2019). Estudio de percepción de los servicios ecosistémicos de la Reserva de Producción de Fauna Chimborazo. Riobamba: Escuela Superior Politécnica de Chimborazo. Retrieved from http://dspace.espoch.edu.ec/handle/123456789/10304

Collaguazo, C. (2019). Valoración económica ambiental del recurso vegetal productivo de la parroquia San Luis, cantón Riobamba, provincia de Chimborazo. Trabajo de titulación presentado para obtar por el grado académico de Ingeniera en Biotecnología Ambiental. Retrieved from http://dspace.espoch.edu.ec/bitstream/123456789/13799/1/236T0476.pdf

Cristeche, E., \& Penna, J. (2008). Métodos de valoración económica de los servicios ambientales. Instituto de Economía y Sociología. doi:ISSN 1851 - 6955

Daniel, T., Muhar, A., Arnberger, A., Aznar, O., Boyd, J., Chan, K., \& Grêt-Regamey, A. (2012). Contributions of cultural services to the ecosystem services agenda. Proceedings of the National Academy of Sciences, 109(23), 8812 - 8819. Retrieved from https://doi.org/10.1073/pnas.1114773109

FAO. (2021). Servicios ecosistémicos y biodiversidad. Retrieved from http://www.fao.org/ecosystem-services-biodiversity/es/

GADPR PUNÍN. (2015). PLAN DE DESARROLLO Y ORDENAMIENTO TERRITORIAL 2015-2019. Retrieved from http://app.sni.gob.ec/snilink/sni/PORTAL_SNI/data_sigad_plus/sigadplusdocumentofinal/06608216400 01_PDOT\%20PUNIN\%202015-2019(reformado)_28-06-2016_22-41-43.pdf

Haines-Young, R., \& Potschin-Young, M. (2018). Revision of the Common International Classification for Ecosystem Services (CICES V5.1): A Policy Brief. One Ecosystem. doi:10.3897/oneeco.3.e27108

INEC. (2021, 05 02). Ecuador en Cifras. Retrieved from https://www.ecuadorencifras.gob.ec/documentos/webinec/Bibliotecas/Fasciculos_Censales/Fasc_Cantonales/Chimborazo/Fasciculo_ Riobamba.pdf

Laurans, Y., Rankovic, A., Billé, R., Pirard, R., \& Mermet, L. (2013). Use of ecosystem services economic valuation for decision making: Questioning a literature 
blindspot. Journal of Environmental Management, 208 - 219. doi:https://doi.org/10.1016/j.jenvman.2013.01.008.

Lemache, K. (2021). Valoración económica ambiental del recurso vegetal de la parroquia Punin. Riobamba: ESPOCH. Retrieved from http://dspace.espoch.edu.ec/bitstream/123456789/14524/1/236T0515.pdf

Life adaptamed. (2018). Qué son los servicios ecosistémicos y cómo podemos beneficiarnos de ellos. Retrieved from https://www.lifeadaptamed.eu/?p=1113

Martín-López, B., Iniesta-Arandia, I., García-Llorente, M., Palomo, I., Casado-Arzuaga, I., García del Amo, D., . . Palacios, I. (2021). Uncovering ecosystem service bundles through social. PloS One. doi:https://doi.org/10.1371/journal.pone.0038970

Mayanquer, P. (2019). Valoración económica ambiental del recurso vegetal productivo de la comunidad de San Nicolás de la parroquia de Licto. Riobamba: Escuela Superior Politécnica de Chimborazo. Retrieved from http://dspace.espoch.edu.ec/handle/123456789/13204

Moreta, M. (2018). Valoración Economica Ambiental del recurso vegetal en la parroquia San Juan, cantón Riobamba. Trabajo de titulación presentado para obtar por el grado académico de Ingeniera en Biotecnología Ambiental, Riobamba. Retrieved from http://dspace.espoch.edu.ec/bitstream/123456789/10556/1/236T0415.pdf

Nieto, C., Añazco, M., Hidrobo, G., Flor, E., Nieto, M., Borrero, J. T., \& Paredes, N. (2016). Informe nacional sobre el estado de la Biodiversidad para la alimentación y la agricultura en el Ecuador. Quito. doi:http://www.fao.org/3/CA3493ES/ca3493es.pdf

Poppenborg, P., \& Koellner, T. (2013). Do attitudes toward ecosystem services determine agricultural land use practices? An analysis of farmers' decision-making in a South Korean watershed. Land Use Policy, 422 - 429. doi:https://doi.org/10.1016/j.landusepol.2012.08.007

Rodriguez, N., Herrera, S., \& Ruiz, R. (2014). Plan de desarrollo turístico comunitario en la parroquia Punín, cantón Riobamba, provincia de Chomborazo. Quito: UCE. Retrieved from http://www.dspace.uce.edu.ec/handle/25000/3065

SIPA. (2020). Sistema de Información Pública Agropecuria. Retrieved from http://sipa.agricultura.gob.ec/

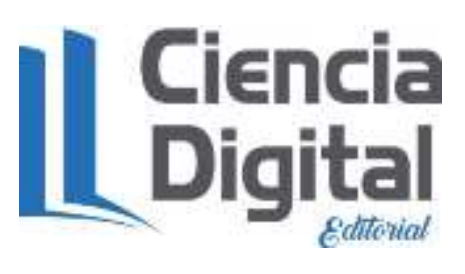




\section{PARA CITAR EL ARTÍCULO INDEXADO.}

Brito Mancero, M. Y., Ruiz Mancero, L. E., \& Lemache Velarde, K. V. (2021). Evaluación económica de los servicios ecosistémicos del recurso vegetal de la parroquia Punin, cantón Riobamba . AlfaPublicaciones, 3(3.1), 202-215. https://doi.org/10.33262/ap.v3i3.1.87

\section{Ciencia \\ Digital \\ Editivial}

El artículo que se publica es de exclusiva responsabilidad de los autores y no necesariamente reflejan el pensamiento de la Revista Alfa Publicaciones.

El artículo queda en propiedad de la revista y, por tanto, su publicación parcial y/o total en otro medio tiene que ser autorizado por el director de la Revista Alfa Publicaciones.
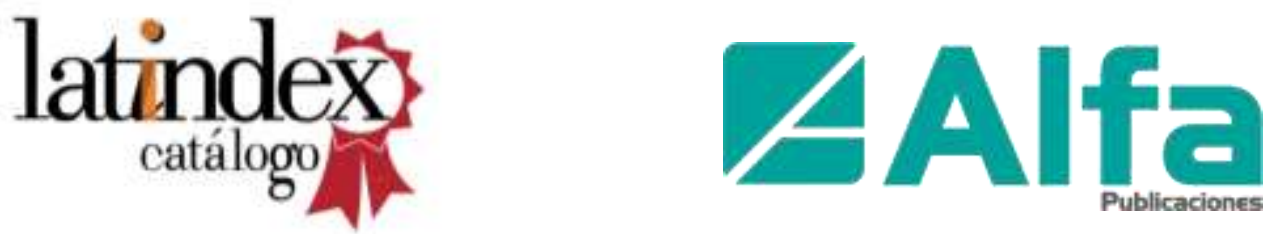